\title{
Subwavelength Bessel beams in wire media
}

\author{
Carlos J. Zapata-Rodríguez ${ }^{1, *}$ and Juan J. Miret ${ }^{2}$ \\ ${ }^{1}$ Department of Optics, University of Valencia, Dr. Moliner 50, 46100 Burjassot, Spain \\ ${ }^{2}$ Department of Optics, Pharmacology and Anatomy, University of Alicante, P.O. Box 99, Alicante, Spain \\ ${ }^{*}$ Corresponding author: carlos.zapata@uv.es
}

\author{
Received September 5, 2013; accepted November 11, 2013; \\ posted November 25, 2013 (Doc. ID 196974); published December 18, 2013
}

\begin{abstract}
Recent progress is emerging on nondiffracting subwavelength fields propagating in complex plasmonic nanostructures. In this paper, we present a thorough discussion on diffraction-free localized solutions of Maxwell's equations in a periodic structure composed of nanowires. This self-focusing mechanism differs from others previously reported, which lie on regimes with ultraflat spatial dispersion. By means of the Maxwell-Garnett model, we provide a general analytical expression of the electromagnetic fields that can propagate along the direction of the cylinder's axis, keeping its transverse waveform unaltered. Numerical simulations based on the finite element method support our analytical approach. In particular, moderate filling fractions of the metallic composite lead to nonresonant-plasmonic spots of light propagating with a size that remains far below the limit of diffraction. (C) 2013 Optical Society of America
\end{abstract}

OCIS codes: $\quad$ (050.2065) Effective medium theory; (160.1245) Artificially engineered materials; (260.1960)

Diffraction theory.

http://dx.doi.org/10.1364/JOSAB.31.000135

\section{INTRODUCTION}

Diffractive broadening is a natural process of wave fields involving an increment of the beam width as it propagates in free space [ $[1,2]$. This diverging process occurs nearer the beam waist as long as it is tighter confined in the transverse direction of propagation [3]. However, such a direct relationship between field localization and diffraction is not so obvious. In the late 1980s, Durnin and co-workers proposed a way to excite Bessel beams (BBs) overcoming diffraction in free space $[\underline{4}, \underline{5}]$. BBs are traveling wave fields in homogeneous dielectric media with a prescribed propagation constant along the direction of propagation, and concurrently its transverse pattern follows a Bessel function of the first kind. Since intensity is confined around a characteristic axis despite diffraction effects, soon they were coined "diffraction-free beams." In fact, the BB beam size is not fully free from diffraction since its FWHM will be greater than half a wavelength. Potential applications of BBs include optical manipulation [6,7], femtosecond laser submicrochannel machining [ $[\underline{8}, \underline{9}]$, and nonlinear microscopy in dense media [10]. Recent progress in theory and applications of BBs may be found in [11] and [12].

Transferring such ideas to optically structured media is strikingly easy to do but still barely explored [13-16]. One reason for this is that undivided attention was given to guided modes due to their key role in telecommunications. Structured or not, a host medium cannot route a wave field in the linear regime by fundamentals. Therefore, nondiffracting beams confined in periodic arrangements might be interpreted as out-of-plane focal waves [17].

If we turn our attention to plasmonic nanostructures, launching of BBs in the form of standing surface modes around a metal-dielectric interface through total internal reflection has been reported [18,19]. Excitation of plasmonic nondiffracting beams propagating with curvilinear trajectory also is possible by means of corrugations and holes drilled on metallic films $[20,21]$. One may ask whether a $1 \mathrm{D}$ projection of the conical angular spectrum of a $2 \mathrm{D}$ diffraction-free solution could yield a surface wave of similar characteristics. In this regard, the answer is negative [22]. Nevertheless, coupled surface plasmons polaritons (SPPs) appearing in complex metamaterials enable a satisfactory approach to generate propagating purely plasmonic BBs. In particular, we theoretically confirmed that a metal-dielectric stratified medium may sustain diffraction-free localized beams, even including losses in the materials [23,24]. Grazing propagation is not sustained by canalization [25], but it depends on the waveform itself. More importantly, the assistance of SPPs leads to subwavelength beam sizes that may be conserved for propagation distances much longer than a wavelength [26].

In this paper, we provide a step forward in the analysis of nondiffracting subwavelength fields sustained in complex plasmonic nanostructures. We will demonstrate the existence of localized nondiffracting beams propagating in a wire medium. Particularly, wire metamaterials are gaining interest and recognition in the metamaterials science [27]. Based on the Maxwell-Garnett model, homogenized composites may sustain hybrid BBs having a two-ring-shaped spatial spectrum. This finding is confirmed by numerical simulations using the finite element method (FEM). Practical limits are reported for excellent agreement with the previous analytical estimations. In particular, we observe that moderate filling fractions of the metallic composite may lead to nonresonant SPPs-driven spots of light propagating with a beam size that remains far below the limit of diffraction. 


\section{DIFFRACTION-FREE BEAMS IN 2D STRUCTURED NONMAGNETIC MEDIA}

We consider a structured material in two dimensions in such a way that the optical properties of the medium change in the $x y$ plane, but they are conserved along the $z$ axis. As a consequence, the relative permittivity of the isotropic inhomogeneous metamaterial is denoted by $\epsilon(x, y)$. Also we assume that monochromatic beam propagation is driven along the axis of symmetry, which is the $z$ axis. We focus on nondiffracting beams, which may be understood as solutions of Maxwell's equations having a transverse waveform that remains unchanged at different $x y$ planes, except maybe by a phase-only term depending on $z$. As a result, we finally may write the magnetic field $\mathbf{H}=\mathbf{h}(x, y) \exp (i \beta z-i \omega t)$, where $\omega=c k_{0}$ is the time-domain frequency and $\beta$ is the on-axis spatial frequency of the wave field.

For convenience, we set the $3 \mathrm{D}$ vector field $\mathbf{h}=\mathbf{h}_{t}+h_{z} \mathbf{z}$, where $\mathbf{h}_{t}=h_{x} \mathbf{x}+h_{y} \mathbf{y}$ includes both transverse components, and $h_{z}$ is considered the on-axis component of the wave field. If the optical system is free of external sources, the wave equation,

$$
\nabla \times\left(\epsilon^{-1} \nabla \times \mathbf{H}\right)=k_{0}^{2} \mathbf{H}
$$

might be further simplified by using the wave field $\mathbf{h}$. For instance, the transverse wave field fulfills a much simpler wave equation:

$$
\left(\epsilon k_{0}^{2}-\beta^{2}+\nabla_{t}^{2}\right) \mathbf{h}_{t}=-\epsilon^{-1} \nabla_{t} \epsilon \times\left(\nabla_{t} \times \mathbf{h}_{t}\right),
$$

which is independent of the on-axis component $h_{z}$. Here $\nabla_{t}=\mathbf{x} \partial_{x}+\mathbf{y} \partial_{y}$ and $\nabla_{t}^{2}=\partial_{x}^{2}+\partial_{y}^{2}$. Equation (2) represents a set of differential equations coupling $h_{x}$ and $h_{y}$ and assuming that $\epsilon$ varies transversally. As a consequence, nondiffracting solutions of the wave in Eq. (2) includes hybrid-polarized solutions. On the other hand, for the on-axis scalar wave field, we use $\mathbf{h}_{t}$ from Eq. (2) and the Gauss' law for magnetism to obtain $h_{z}=(i \beta)^{-1} \nabla_{t} \overline{\mathbf{h}}_{t}$.

In this study, we consider wires of radius $r$, whose permittivity is denoted by $\epsilon_{m}$. For the sake of clarity, first we disregard losses in the metal and therefore $\epsilon_{m}<0$. We assume a periodic squared distribution of these wires in a way that $a$ stands for the lattice period along the $x$ axis and the $y$ axis, as shown in Fig. 1. Note that $a \geq 2 r$. The host medium has a positive dielectric constant $\epsilon_{d}$. In our system, a given wire axis is parallel to the unit vector $\mathbf{z}$; therefore we set $\epsilon(x, y)=\epsilon_{m}$ in the metallic rods and $\epsilon(x, y)=\epsilon_{d}$ in the host medium. Finally, we assume that the monochromatic beam propagates along the wires without defocusing.

\section{BESSEL BEAMS IN EFFECTIVE UNIAXIAL MEDIA}

We may simplify our problem by considering the effective medium approximation (EMA) [28-30]. Under this approach, the wire metamaterial is modeled as an anisotropic medium. In this case, the metal-dielectric compound behaves like a uniaxial crystal whose optic axis is oriented along the $z$ axis. As a consequence, $\epsilon(x, y)$ is transformed into a dyadic permittivity $\bar{\epsilon}=\epsilon_{\perp}(\mathbf{x} \otimes \mathbf{x}+\mathbf{y} \otimes \mathbf{y})+\epsilon_{\|} \mathbf{z} \otimes \mathbf{z}$. Here, the parameters,

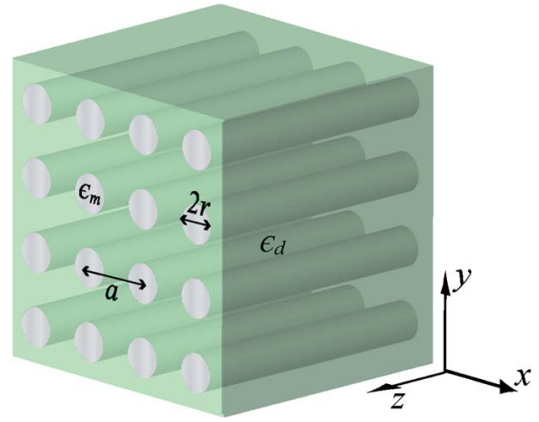

Fig. 1. Periodic array of nanowires made of a metal with dielectric constant $\epsilon_{m}$, distributed in a squared lattice, and hosted in a dielectric medium with permittivity $\epsilon_{d}$. The radius of the wires is $r$, and the lattice constant is $a$. Beam propagation is driven along the wire's axis, which is the $z$ axis.

$$
\begin{gathered}
\epsilon_{\perp}=\frac{\left[(1+f) \epsilon_{m}+(1-f) \epsilon_{d}\right] \epsilon_{d}}{(1-f) \epsilon_{m}+(1+f) \epsilon_{d}}, \\
\epsilon_{\|}=f \epsilon_{m}+(1-f) \epsilon_{d},
\end{gathered}
$$

are taken from the Maxwell-Garnett (MG) theory [31], being $f=\pi r^{2} / a^{2}$ the metal filling fraction in the wire medium.

We point out that the MG theory can be substituted by some other homogenization approaches in the long-wavelength limit, which can be found elsewhere, to elucidate the effective refractive indices of the structure [32]. Nevertheless, we will show that the MG theory provides satisfactory results on the condition that the lattice period, $a$, is significantly lower than the penetration depth in the conductor, $c / \omega_{p}$, being $\omega_{p}$ the plasma frequency of the metal. In turn, $a$ becomes much lower than the wavelength $\lambda_{0}=2 \pi / k_{0}$.

Note that nondiffracting beams propagating in uniaxial anisotropic media have already been analyzed, assuming they travel along (also orthogonally to) the optical axis of the crystal [33]. In the present section, we review the main attributes of these BBs.

\section{A. TE Bessel Beams}

Under the assumptions taken from the EMA and given above, $h_{z}$ satisfies the Helmholtz equation in two dimensions,

$$
\left(k_{t}^{2}+\nabla_{t}^{2}\right) h_{z}=0
$$

where the in-plane wavenumber of the wave field, $k_{t}$, is typified here as

$$
k_{t}^{o}=\sqrt{\epsilon_{\perp} k_{0}^{2}-\beta^{2}},
$$

provided that $\epsilon_{\perp}>0$ and $\beta \leq \sqrt{\epsilon_{\perp}} k_{0}$. Note that this is a wavenumber, $k_{t}^{o}$, corresponding to ordinary waves (o waves) propagating in a uniaxial crystal of permittivity $\bar{\epsilon}$. In other words, these wave fields are transverse electric (TE) modes, since the on-axis component of the electric field vanishes. Solutions using Bessel functions come out naturally by setting $\nabla_{t}^{2}$ in a cylindrical coordinate system, which is $\nabla_{t}^{2}=\partial_{r}^{2}+r^{-1} \partial_{r}+r^{-2} \partial_{\phi}^{2}$. Solving the Helmholtz wave equation for the $o$ waves yields 


$$
h_{z}^{o}(r, \phi)=\frac{k_{t}^{o}}{\beta} \sum_{m=-\infty}^{\infty} A_{m} \psi_{m}^{o}(r, \phi),
$$

where $A_{m}$ denotes a complex-valued constant,

$$
\psi_{m}^{o}(r, \phi)=\exp (i m \phi) J_{m}\left(k_{t}^{o} r\right),
$$

and $J_{m}(\cdot)$ is a Bessel function of the first kind. Equation (7) gives a complete solution provided that $h_{z}^{o}$ does not diverge at $r=0$.

Apart from that, it is useful to express the Bessel wave field in the form of a plane-wave Fourier expansion. In particular,

$$
\psi_{m}^{o}(r, \phi)=(-i)^{m} \int_{0}^{2 \pi} \exp (i m \theta) \exp \left(i \mathbf{k}_{t}^{o} \mathbf{r}\right) \mathrm{d} \theta
$$

Note that a point $\mathbf{r}=\mathbf{x} r \cos \phi+\mathbf{y} r \sin \phi$ is set in the polar coordinate system. Equation (9) stands for a equienergetic superposition of 2D plane waves, modulated azimuthally by a phase-only linear term, whose wave vector $\mathbf{k}_{t}^{o}=\mathbf{x} k_{t}^{o} \cos \theta+$ $\mathbf{y} k_{t}^{o} \sin \theta$ has the wavenumber $k_{t}^{o}$ given in Eq. (6). Since the magnetic field $\mathbf{H}$ includes the on-axis spatial frequency $\beta$, the TE BB may be considered a conical distribution of $o$ waves whose revolution axis is set along the $z$ axis, as shown in Fig. 2(a). The angular spectrum of $h_{z}^{o}, P_{2}(\theta)$, may vary in phase and in amplitude, as illustrated in Fig. 2(b).

The transverse components of the magnetic field may be obtained by means of the Gauss' law for magnetism. They are simply written as

$$
\mathbf{h}_{t}^{o}=-\frac{1}{2} \sum_{m=-\infty}^{\infty} A_{m}\left[i\left(\psi_{m+1}^{o}-\psi_{m-1}^{o}\right) \mathbf{x}+\left(\psi_{m+1}^{o}+\psi_{m-1}^{o}\right) \mathbf{y}\right]
$$

We point out that the transverse field of the TE modes also satisfies the following wave equation:

$$
\left(\epsilon_{\perp} k_{0}^{2}-\beta^{2}+\frac{\epsilon_{\perp}}{\epsilon_{\|}} \nabla_{t}^{2}\right) \mathbf{h}_{t}=i \beta\left(1-\frac{\epsilon_{\perp}}{\epsilon_{\|}}\right) \nabla_{t} h_{z}
$$

which may be applied broadly, not only for $o$ waves.

\section{B. TM Bessel Beams}

Nontrivial solutions of Maxwell's equations exist involving $h_{z}=0$, which is the transverse magnetic (TM) mode. These solutions are associated with extraordinary waves ( $e$ waves) in the effective uniaxial medium. In this case, the transverse spatial frequency $k_{t}$ follows the expression
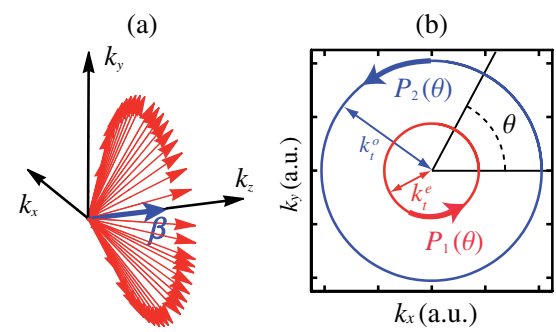

Fig. 2. (a) Conical distribution of wave vectors in agreement with the plane-wave Fourier expansion of BBs in effective uniaxial media. (b) Annular-shaped spectrum of TM BBs, $P_{1}(\theta)$, and TE BBs, $P_{2}(\theta)$. The arrows illustrate the angular dependence of the spectra.

$$
k_{t}^{e}=\sqrt{\epsilon_{\|} k_{0}^{2}-\beta^{2} \epsilon_{\|} / \epsilon_{\perp}}
$$

as may be deduced straightforwardly from Eq. (11) by means of the substitution $\nabla_{t}^{2} \rightarrow-k_{t}^{2}$ and setting $h_{z}=0$. Note that $k_{t}^{e}=\sqrt{\epsilon_{\|} / \epsilon_{\perp}} k_{t}^{o}$; that is, $k_{t}^{e}$ differs from $k_{t}^{o}$ in the uniaxial medium as illustrated in Fig. 2(b). In addition, $k_{t}^{e}$ and $k_{t}^{o}$ exist in the domain $0 \leq \beta \leq \sqrt{\epsilon_{\perp}} k_{0}$ for all positive permittivities.

Interestingly, if $\epsilon_{\|}<0<\epsilon_{\perp}$ characterizing an hyperbolic metamaterial [34], $o$ waves are purely evanescent, but $k_{t}^{e}$ is unbounded in the domain $\sqrt{\epsilon_{\perp}} k_{0}<\beta$. Otherwise, if $\beta<\sqrt{\epsilon_{\perp}} k_{0}$, $e$ waves cannot propagate and decay exponentially in the metamaterial. In conclusion, the hyperbolic regime prevents the hybridization of the polarization states of BBs. A thorough analysis of spatial dispersion of $o$ waves and $e$ waves in the wire medium will be given below.

Dispersion relation for TM modes is also inferred from the wave equation corresponding to the electric field, $\mathbf{E}=\mathbf{e}(x, y) \exp (i \beta z-i \omega t)$. In particular, we have

$$
\left(\epsilon_{\perp} k_{0}^{2}-\beta^{2}+\frac{\epsilon_{\perp}}{\epsilon_{\|}} \nabla_{t}^{2}\right) e_{z}=0
$$

for the on-axis component of the electric field. Formally, such a wave equation is the same as that given in Eq. (5), after a convenient substitution $k_{t}^{o} \rightarrow k_{t}^{e}$ and $h_{z} \rightarrow e_{z}$, thus exhibiting the same typology of solutions. Therefore we may write

$$
e_{z}^{e}(r, \phi)=\frac{k_{t}^{e}}{\beta} \sum_{m=-\infty}^{\infty} B_{m} \psi_{m}^{e}(r, \phi),
$$

where $B_{n}$ stands for a complex-valued constant and $\psi_{m}^{e}(r, \phi)=\exp (i m \phi) J_{m}\left(k_{t}^{e} r\right)$. Now the magnetic field of the associated Bessel waves is given by

$$
\mathbf{h}_{t}^{e}=+\frac{1}{2 Z_{B}} \sum_{m=-\infty}^{\infty} B_{m}\left[\left(\psi_{m+1}^{e}+\psi_{m-1}^{e}\right) \mathbf{x}+i\left(-\psi_{m+1}^{e}+\psi_{m-1}^{e}\right) \mathbf{y}\right]
$$

where $Z_{B}=Z_{0} \beta / \epsilon_{\|} k_{0}$ is the intrinsic impedance of the $\mathrm{BB}$, and $Z_{0}$ is the impedance of free space. From the Ampere's law, we finally confirm that $\nabla_{t} \times \mathbf{h}_{t}=-i \mathbf{z} e_{z} \beta / Z_{B}$.

\section{Spatial Spectrum of Bessel Beams}

A relevant feature of the $\mathrm{BB}$ waveform is in relation with its spatial spectrum. From a mathematical point of view, the transverse spatial spectrum of the wave field is retrieved by applying the 2D Fourier transform (2D-FT) to its components. By definition, the 2D-FT of a function $g(\mathbf{r})$ is derived from

$$
F\{g\}\left(\mathbf{k}_{t}\right)=\iint g(\mathbf{r}) \exp \left(-i \mathbf{k}_{t} \mathbf{r}\right) \mathrm{d}^{2} \mathbf{r} .
$$

By means of the Bessel function closure in Eq. [35], we arrive at the following result:

$$
F\left\{\psi_{m}(r, \phi)\right\}=(2 \pi)^{2} k_{t}^{-1}(-i)^{m} \exp (i m \theta) \delta\left(k_{t}-\left|\mathbf{k}_{t}\right|\right),
$$

where the frequency $\mathbf{k}_{t}=\mathbf{x} k_{x}+\mathbf{y} k_{y}$ is set in the polar coordinate system, $\left(\left|\mathbf{k}_{t}\right|, \theta\right)$, and $\delta$ is the Dirac delta function. This is 
also straightforwardly deduced from Eq. (9). Note that the spectral content of the function $\psi_{m}$ is localized along an annulus of radius $\left|\mathbf{k}_{t}\right|=k_{t}$, independently of its order $m$, as illustrated in Fig. 2(b).

Let us now consider the 2D spatial spectrum of the magnetic fields, $\mathbf{h}_{t}^{o}$ and $\mathbf{h}_{t}^{e}$, given in Eqs. (10) and (15), respectively. Every component of these wave fields in the Fourier domain consists of a single ring, possibly varying with the angular coordinate. In other words, the isofrequency curve exhibits circular symmetry; this is in contrast to transverse spectra that can be found in arrangements studied elsewhere [14,15]. The axisymmetry of the modal fields is caused by the homogenization of the wire medium, which is valid in the longwavelength approach. Otherwise, nonlocal effects will arise leading to fine wave structures near the metallic elements, as we will see below. Finally, since the value of $k_{t}^{o}$ differs from $k_{t}^{e}$ given a propagation constant $\beta$, hybrid-polarized solutions involving the simultaneous contribution of $\mathbf{h}_{t}^{o}$ and $\mathbf{h}_{t}^{e}$ would provide a spatial spectrum having a bi-annular pattern, as depicted in Fig. 2(b).

\section{SPATIAL DISPERSION IN WIRE MEDIA}

In the EMA, the dyadic permittivity $\bar{\epsilon}$ of the effective uniaxial medium is governed by the permittivities of the dielectric and conducting materials in the composite, but also depends strongly on the metal filling fraction $f$. This effect may lead to dispersion regimes of a different nature, either elliptic dispersion or hyperbolic dispersion, by simply tuning the filling fraction of the metal. To illustrate this effect, we will consider $\epsilon_{d}<\left|\operatorname{Re}\left(\epsilon_{m}\right)\right|$ valid for visible and lower frequencies. For numerical purposes, we particularly introduce silver wires in an alumina matrix. The permittivities of silver and alumina at the wavelength $\lambda_{0}=700 \mathrm{~nm}$ are $\epsilon_{\mathrm{Ag}}=-20.4$ (neglecting losses) and $\epsilon_{\mathrm{Al}_{2} \mathrm{O}_{3}}=3.1$, respectively, which are taken from experimental data [36].

Figure 3 shows the effective permittivities $\epsilon_{\|}$and $\epsilon_{\perp}$ given by Eqs. (3) and (4) for our silver-alumina composite. For low filling fractions, both permittivities are positive, satisfying $\epsilon_{\|}<\epsilon_{d}<\epsilon_{\perp}$. As long as $f$ increases, $\epsilon_{\perp}$ grows significantly; contrarily $\epsilon_{\|}$decreases in linear proportion to $f$. Therefore, birefringence is negative in the wire medium, and it varies vigorously along with $f$ in the elliptic regime. In fact, $\epsilon_{\|}>0$ for

$$
0 \leq f<\frac{\epsilon_{d}}{\epsilon_{d}-\epsilon_{m}}
$$

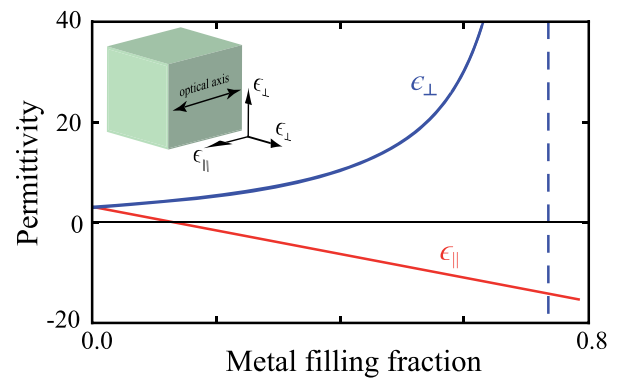

Fig. 3. Variation of $\epsilon_{\perp}$ and $\epsilon_{\|}$in terms of the filling fraction, by using the Maxwell-Garnett model, for silver wires hosted in alumina at $\lambda_{0}=700 \mathrm{~nm}$. Inset: Schematic illustration of the anisotropic medium that homogenizes the wire plasmonic crystal of Fig. 1.
In the numerical simulation, Eq. (18) is rewritten as $0 \leq f<0.132$, where $f$ is bounded by a relatively low value of the filling fraction, which is caused by the relatively low ratio $\epsilon_{d} /\left|\epsilon_{m}\right|$. As a consequence, the on-axis permittivity becomes negative for moderate and high filling fractions. On the other hand, $\epsilon_{\perp}$ is maintained positive up to much higher filling fractions:

$$
0 \leq f<\frac{\epsilon_{m}+\epsilon_{d}}{\epsilon_{m}-\epsilon_{d}}
$$

This yields $0 \leq f<0.737$ for our metal-dielectric composite. Figure 3 shows that $\epsilon_{\perp}$ may reach extremely high positive values in the interval $0.132 \leq f<0.737$, where $\epsilon_{\|}$is negative, leading to hyperbolic dispersion. Note that the filling fraction is limited by its maximum value, $f_{\max }=\pi / 4 \approx 0.78$, which occurs when adjacent wires are put into contact in the squared arrangement, $a=2 r$. Near this upper bound, the wire medium behaves like an effective anisotropic conductor. Finally, it should be mentioned that there is an upper limit on the filling fraction imposed by the applicability of the Maxwell-Garnett formula, which is known to be valid for lower filling fractions only [37].

As mentioned previously, BBs driven by $o$ waves [see Eq. (10)] can exist only if $\beta<\sqrt{\epsilon_{\perp}} k_{0}$. Instead, the singular dispersion regimes exhibited by $e$ waves allow the existence of BBs with a propagation constant that ideally may take any positive value, $0 \leq \beta<\infty$. However, it depends strongly on the sign of $\epsilon_{\|}$, as illustrated in Fig 4 . For instance, if $\epsilon_{\|}>0$ then $\beta<\sqrt{\epsilon_{\perp}} k_{0}$ for the existence of extraordinary waves. Moreover, since $k_{t}^{e}=\sqrt{\epsilon_{\|} / \epsilon_{\perp}} k_{t}^{o}$ and birefringence is negative $\left(\epsilon_{\|} / \epsilon_{\perp}<1\right)$ in the elliptic regime, TE BBs will be tighter confined than TM modes. On the contrary, solutions of Maxwell's equations involving $\beta>\sqrt{\epsilon_{\perp}} k_{0}$ are consistent with EMA provided that $\epsilon_{\|}<0$ (and obviously $\epsilon_{\perp}>0$ ). Assuming a sufficiently high value of $\beta$, we infer that $k_{t}^{e} \approx \beta \sqrt{\left|\epsilon_{\|}\right| / \epsilon_{\perp}}$ for TM polarization, which is an asymptotic behavior included in Fig 4. In this case $k_{0} \ll k_{t}^{e}<\beta$, and therefore the spot size of the BB clearly surpasses the limit imposed by diffraction.

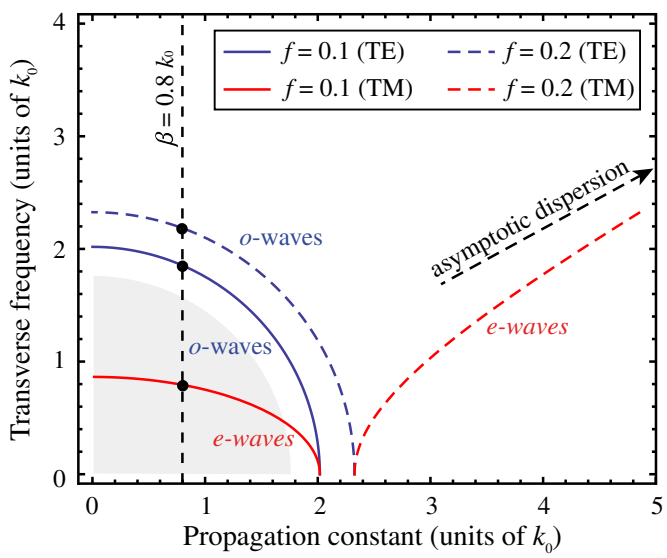

Fig. 4. Isofrequency curve in the $\beta k_{t}$ plane for TE and TM modes propagating in an Ag-wire medium hosted in alumina at $\lambda_{0}=700 \mathrm{~nm}$. Equations (6) and (12) are plotted for a filling fraction $f=0.1\left(\epsilon_{\perp}=4.07\right.$ and $\left.\epsilon_{\|} \overline{=} 0.75\right)$ and for $f=0.2$ where $\epsilon_{\perp}=5.41$ and $\epsilon_{\|}=-1.6$. We also include a shaded area bounded by the isofrequency curve of $\mathrm{Al}_{2} \mathrm{O}_{3}(f=0)$ wherein diffraction-limited waves are included. Black dots represent frequencies used in Fig. $\underline{5}$ and succeeding figures. 
In practical terms, however, it is much easier to excite BBs with moderate and low propagation constants. We point out that deeply subwavelength nondiffracting beams also may propagate with low $\beta$ under TE polarization within the regime of indefinite permittivity. Specifically, if $\epsilon_{\perp}$ takes high values, happening near $f_{\max }$, then $k_{t}^{o} \approx \sqrt{\epsilon_{\perp}} k_{0} \gg k_{0}$.

At this point, we emphasize that BBs propagates with no distortion of its transverse pattern precisely due to the singular characteristics of the spatial distribution of this wave field. This self-focusing mechanism differs from canalization (also known as self-guiding), which lies in the existence of a flat dispersion curve within a broad spatial spectrum. For TM modes, the canalization regime occurs when $\epsilon_{\|} \rightarrow \infty$, leading to $\beta=\sqrt{\epsilon_{\perp}} k_{0}$ [25]. Based on the EMA, a wire medium cannot become an anisotropic crystal with an infinite permittivity along the anisotropy axis. Nevertheless, canalization in wire media has been reported elsewhere [38]. In this case, selfguiding can be derived from the strong nonlocality observed in microwaves, but it cannot be realized at an optical range where metals lose their conducting properties.

\section{NUMERICAL EXAMPLES}

We have numerically simulated wave propagation of BBs directed along the effective optic axis of a silver-alumina wire medium at a moderate propagation constant, $\beta=0.8 k_{0}$, being $\lambda_{0}=700 \mathrm{~nm}$. That is $\beta=7.18 \mu \mathrm{m}^{-1}$. In Figs. 5(a) and 5(b), we plot $\left|h_{x}^{e}\right|^{2}$ and $\left|h_{x}^{o}\right|^{2}$ taken from Eqs. (15) and (10), respectively. Specifically we considered an $\mathrm{Ag}-\mathrm{Al}_{2} \mathrm{O}_{3}$ composite with a filling fraction $f=0.1$. In this case, the MG model provides the effective permittivities $\epsilon_{\perp}=4.07$ and $\epsilon_{\|}=0.75$. Since both permittivities are positive, elliptic dispersion governs $e$ waves.

For convenience, first we used $B_{1}=Z_{B}$ and $B_{-1}=Z_{B}$ (in arbitrary units) to evaluate the wave field $h_{x}^{e}$. The remaining coefficients $B_{m}$ are zero. This BB is characterized by a transverse spatial frequency $k_{t}^{e}=7.12 \mu \mathrm{m}^{-1}$. As a result, the central hot spot of the BB shows an FWHM of $454 \mathrm{~nm}$ along the $x$ axis. In the perpendicular direction, the beam slightly compresses leading to a certain transverse anisotropy, as is evident in Fig. 5(a), which is not related with the effective medium but the asymmetry of the field distribution itself. We conclude that the BB width is on the order of the wavelength. In this case, light concentration around the beam axis is weaker than that obtained in an all-dielectric compound ( $f=0)$, as highlighted in Fig. 4.

On the other hand, the amplitudes $A_{1}=-i$ and $A_{-1}=i$ (also in arbitrary units) are set for the evaluation of the field $h_{x}^{o}$. For TE modes, we estimated $k_{t}^{o}=16.6 \mu \mathrm{m}^{-1}$ for $f=0.1$, leading to superresolving hot spots of $110 \mathrm{~nm}$ FWHM on
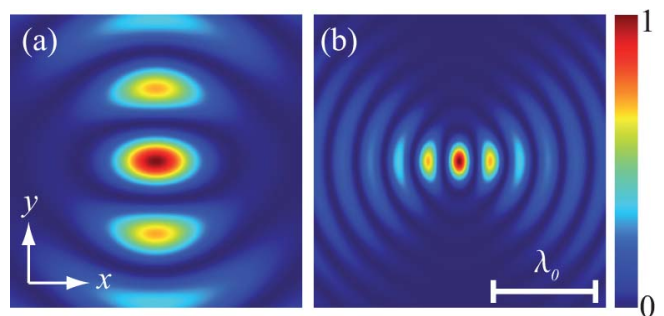

Fig. 5. Wave field $\left|h_{x}\right|^{2}$ for BBs associated with (a) $e$ waves and (b) $o$ waves propagating in a homogenized $\mathrm{Ag}_{-} \mathrm{Al}_{2} \mathrm{O}_{3}$ medium $(f=0.1)$ provided that $\beta=0.8 k_{0}$. We represent the fields for $B_{1}=Z_{B}=B_{-1}$ and $A_{1}=-i=-A_{-1}$, respectively. Box dimensions of the contour plots are $2 \mu \mathrm{m} \times 2 \mu \mathrm{m}$. the $x$ direction. Contrarily to $e$ waves, this is clearly a subwavelength effect. In this case, additionally, wave confinement is lower along the $y$ axis, as shown in Fig. 5(b).

For the sake of completeness, let us write the $x \overline{\text { component }}$ of the vector field $\mathbf{h}_{t}^{o}$ plotted in Fig. $\underline{5(b)}$ in terms of a plane-wave Fourier expansion. Using Eqs. (ㅁ) and (10), we finally have

$$
h_{x}^{o}(r, \phi)=\int_{0}^{2 \pi} P_{2}(\theta) \exp \left(i \mathbf{k}_{t}^{o} \mathbf{r}\right) \mathrm{d} \theta
$$

where

$$
P_{2}(\theta)=1+\cos 2 \theta,
$$

as expressed in arbitrary units. Therefore, the conical distribution of TE plane waves leading to the BB shown in Fig. 5(b) is not flat; the variation of this angular spectrum is apodized by the wave function $P_{2}(\theta)$. Note that such an angular distribution is different, specifically

$$
P_{1}(\theta)=1-\cos 2 \theta,
$$

for the example given above in TM polarization [see Fig. 5(a)].

Next we consider an increase of the filling fraction up to $f=0.2$, still maintaining the propagation constant $\beta$ fixed. As expected, $e$ waves cannot be observed due to its hyperbolic dispersion regime, where $\epsilon_{\|}=-1.6$, and the relatively high value of $\epsilon_{\perp}=5.41$ (see Fig. 4). Precisely, the latter leads to TE BBs with ultra-high spatial frequency, namely $k_{t}^{o}=19.6 \mu \mathrm{m}^{-1}$. Although not represented graphically, the field $\left|h_{x}^{o}\right|^{2}$ has the same waveform as shown in Fig. $\underline{5(\mathrm{~b})}$ but resized in inverse proportion to its transverse wavenumber $k_{t}^{o}$. As a result, the central peak has an FWHM as short as $93 \mathrm{~nm}$ along the $x$ axis. This fact demonstrates that our BBs potentially feature deep-subwavelength hot spots under practical standards.

\section{FULL-WAVE MODES WITH TRANSVERSE CONFINEMENT}

Let us consider solving numerically the Maxwell's equations, equivalently to find the solutions of the wave in Eq. (2) in order to obtain the exact wave fields that are associated with the nondiffracting beams analyzed above. Wave localization will be given at the point $\mathbf{r}=(0,0)$ in the transverse section of the wire medium shown in Fig. 1 . In the majority of numerical simulations, the beam axis will be set at the center of a silver wire. However, we will also examine the differences found when relocating the beam axis at midpoint between two adjacent wires.

Next we will verify the validity of the above analytical approach obtained from the EMA for the periodic squared array of nanowires. For this purpose, we conveniently perform a modal expansion. According to the Floquet-Bloch theorem, the magnetic field of a wave mode in a $2 \mathrm{D}$ periodic medium with invariant spatial frequency $\beta$ along the $z$ axis may be written in the form

$$
\mathbf{H}_{\mathbf{k}_{B}}=\mathbf{h}_{\mathbf{k}_{B}}(\mathbf{r}) \exp \left(i \mathbf{k}_{B} \mathbf{r}\right) \exp (i \beta z-i \omega t)
$$

where $\mathbf{h}_{\mathbf{k}_{B}}(x, y)$ is a field with the same periodicity of the medium, and $\mathbf{k}_{B}=\left(k_{B x}, k_{B y}\right)$ is the in-plane Bloch $\mathbf{k}$ vector. 
For convenience, we impose the complex vector function to have a given normalization, namely

$$
\int_{-a / 2}^{a / 2} \mathrm{~d} x \int_{-a / 2}^{a / 2} \mathrm{~d} y\left|\mathbf{x} \cdot \mathbf{h}_{\mathbf{k}_{B}}(x, y)\right|^{2}=1,
$$

and also that $\mathbf{x} \cdot \mathbf{h}_{\mathbf{k}_{B}}$ will take a real and positive value at the origin. Nondiffracting beams propagating in wire media may be expressed as a linear combination of the wave modes given in Eq. (23). In particular, Bessel-like solutions will be reproduced following a Fourier expansion in the terms of Eqs. (9) and (20).

We focus on solving $\mathbf{k}_{B}$ and $\mathbf{h}_{\mathbf{k}_{B}}(\mathbf{r})$ provided that the propagation constant $\beta$ is a parameter given in our problem. To that end, we used a commercial FEM package (COMSOL Multiphysics). In particular, a routine was programmed in the $\mathrm{RF}$ module that allows us to obtain the Bloch modes $\mathbf{h}_{\mathbf{k}_{B}}$ for a fixed value of $\beta$. Along these lines, we found the complete set of pairs $\left(k_{B x}, k_{B y}\right)$, and their corresponding wave functions $\mathbf{h}_{\mathbf{k}_{B}}$, satisfying the Maxwell's equations for the default $\beta$. This procedure let us depict the spatial spectrum in the $\mathbf{k}_{B}$ plane; in other words, the isofrequency curve provided the on-axis frequency $\beta$. If the EMA remains valid, we expect that the isofrequency curve draws two branches of circular symmetry and with radii $k_{t}^{o}$ and $k_{t}^{e}$.

First we consider a silver-alumina wire medium with filling fraction $f=0.1$, where the diameter of the $\mathrm{Ag}$ wires is $d=2 r=5 \mathrm{~nm}$. In this case, the lattice period is $a=14 \mathrm{~nm}$. Note that the wire diameter is substantially lower than the penetration depth of silver, which is estimated as $24 \mathrm{~nm}$ $\left(\omega_{p}=12.9 \mathrm{fs}^{-1}\right)$, and therefore the metal becomes transparent. Consequently, this configuration is expected to give accurate results in agreement with the EMA. Using our routine based on the FEM for $\beta=0.8 k_{0}$, we obtained the isofrequency curve shown in Fig. 6(a). In this case, the isofrequency curve has two branches approaching circles of radius $k_{B 1}=$ $7.16 \mu \mathrm{m}^{-1}$ and $k_{B 2}=16.6 \mu \mathrm{m}^{-1}$. We point out that these values come very near $k_{t}^{e}$ and $k_{t}^{o}$, respectively, predicted by the EMA for the TM and the TE polarization. The changes in the radial coordinate for different azimuthal angles are negligible within its corresponding branch. In Fig. 6(b), we also represent the field intensity $\left|\mathbf{x} \cdot \mathbf{h}_{\mathbf{k}_{B}}\right|^{2}$ of Bloch modes in a unit cell for three different $\mathbf{k}_{B}$ vectors. A dipolar distribution is evident, occurring also for $\left|\mathbf{y} \cdot \mathbf{h}_{\mathbf{k}_{P}}\right|^{2}$ (not shown in the figure). For modes in the branch $q=1$ (of lower spatial frequency), the orientation of the field distribution is conserved at different angles $\theta$. On the contrary, its orientation follows that of the spatial frequency $\mathbf{k}_{B}$ for the second branch, $q=2$. However, the variations of the field intensity are small and localized very near the wire boundaries.

In view of these results, we previse the formation of nondiffracting beams with Bessel waveforms like those shown in Figs. $5(\mathrm{a})$ and $5(\mathrm{~b})$. Hereunto we will proceed similarly with the plane-wave Fourier expansion given in Eqs. (9) and (20). This integral transformation sets forward a BB waveform by means of a complete set of plane waves. In the periodic wire medium, however, Bloch modes will play such a role. For the TM BB shown in Fig. 5(a), we would obtain an equivalent waveform by properly superposing Bloch modes with wave vectors $\mathbf{k}_{B 1}$, which lie on the spectral branch near the annulus of radius $k_{B 1}$. Also we used the second branch with Block (a)

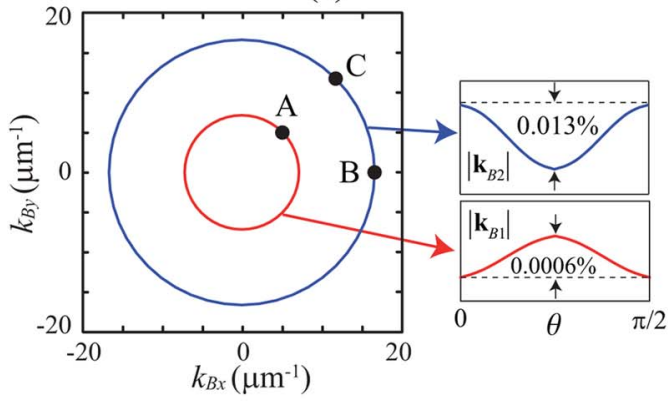

(b)

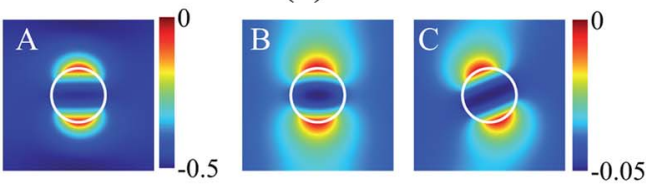

(c)

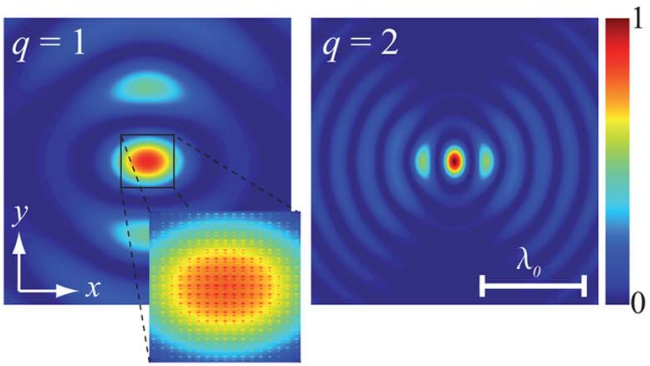

Fig. 6. (a) Isofrequency curve for the Ag-wire medium hosted in $\mathrm{Al}_{2} \mathrm{O}_{3}(f=0.1$ and $d=5 \mathrm{~nm})$ assuming $\beta=0.8 k_{0}$. The insets illustrate the angular dependence of the two branches. (b) Normalized intensity of the $x$ component of the field $\mathbf{h}_{\mathbf{k}_{B}}$ for Bloch modes pointed as $\mathrm{A}, \mathrm{B}$, and $\mathrm{C}$ in the isofrequency curve. Units are set in $\mathrm{dB}$. (c) Field intensity $\left|h_{x q}\right|^{2}$ resulting from Eq. (25) for nondiffracting beams associated with $e$ waves $(q=1)$ and $o$ waves $(q=2)$.

wave vectors $\mathbf{k}_{B 2}$, placed close to the ring of radius $k_{B 2}$, in order to reproduce the $\mathrm{BB}$ in TE polarization sustained in the EMA. The $x$ component of the wave field $\mathbf{h}_{t}$ for both BBs are evaluated numerically by

$$
h_{x q}(\mathbf{r})=\int_{0}^{2 \pi}\left[\mathbf{x} \cdot \mathbf{h}_{\mathbf{k}_{B q}}(\mathbf{r})\right] P_{q}(\theta) \exp \left(i \mathbf{k}_{B q} \mathbf{r}\right) \mathrm{d} \theta,
$$

where $q=1$ is used to reproduce a wave field in association with the TM BB, and $q=2$ will correspond to the TE BB. Note that this integral transform includes an infinite set of Bloch modes. For numerical purposes, however, we have chosen a finite subset of modes that are evenly spaced along with the angular coordinate $\theta$. Finally, depending of the case, we found that the resulting nondiffracting beams have a small but not negligible contribution of the electric/magnetic field along the $z$ axis, thus not a single one of the BBs is either purely TE or TM polarized.

In Fig. 6(c), we plot the intensity of the wave function $h_{x q}$, for $q=1$ and $q=2$, resulting from the superposition of Bloch modes given in Eq. (25). The envelope of the fields is in excellent agreement with the wave fields derived from our analytical approach and shown in Figs. 5(a) and 5(b). However, abrupt changes around the metallic cylinders are evident. For $q=1$, we estimate that the nondiffracting beam has an FWHM as short as $389 \mathrm{~nm}$ along the $x$ axis, which is slightly below the 
(a)

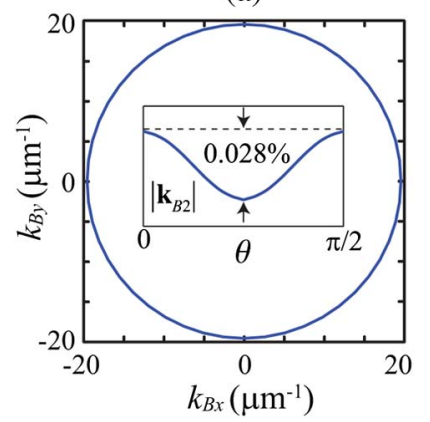

(b)

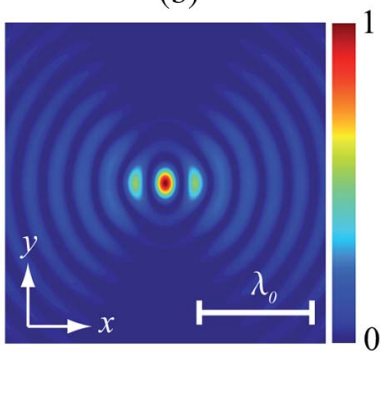

Fig. 7. (a) Isofrequency curve for a metallic compound of $f=0.2$ and a wire diameter of $d=5 \mathrm{~nm}$. Again the propagation constant is $\beta=0.8 k_{0}$. The inset illustrates the angular dependence of the single branch. (b) Wave field $\left|h_{x 2}\right|^{2}$ corresponds to a nondiffracting beam that gets connected with the TE BB in the EMA.

FWHM of the TM BB propagating in the effective medium [see Fig. $\underline{5(\mathrm{a})]}$ ]. On the other hand, $q=2$ leads to a FWHM of $108 \mathrm{~nm}$ as derived from Fig. 6(c), which continues to be clearly subwavelength and extremely close to the data obtained from the EMA [see Fig. 5(b)].

Next we considered an increase of the filling fraction up to $f=0.2$, maintaining the propagation constant $\beta=0.8 k_{0}$ and the diameter $d=5 \mathrm{~nm}$. Now the lattice period is $a=9.9 \mathrm{~nm}$. From our numerical FEM simulations, shown in Fig. 7(a), we observe that only a single ring of radius $k_{B 2}=19.6 \overline{\mu \mathrm{m}^{-1}}$ remains, which is in practice the same frequency estimated by the EMA, which is $k_{t}^{o}$. Again the variation of the radius is small; however, these deviations are approximately doubled in magnitude when they are compared with the case $f=0.1$. The FWHM of the central hot spot measured along the $x$ axis is $89 \mathrm{~nm}$ [see Fig. 7(b)], which is close to $93 \mathrm{~nm}$ measured from Fig. $\underline{5(b)}$.

\section{EFFECTS OF WIRE DIAMETER AND METAL DISSIPATION}

The EMA proves good agreement with FEM simulations provided that the wire diameter remains further down the penetration depth in the metal. Otherwise, severe deviations may arise. In Fig. 8(a), we show the isofrequency curve of a metal-dielectric metamaterial composed of silver wires of diameter $d=50 \mathrm{~nm}$, assuming that the beam propagation constant remains $\beta=0.8 k_{0}$. In general, curvature varies at different orientations of the Bloch wave vector $\mathbf{k}_{B}$. For all practical purposes, this effect might be considered of minor importance, and thus we would expect the formation of waveforms that resemble the fields derived from the EMA. The most outstanding fact of the isofrequency curves is that the Bloch vector $\mathbf{k}_{B}$ for the branch $q=1(f=0.1)$ increases in radius significantly. As a result, the nondiffracting beams resulting from Eq. (25) will display a central spot of reduced FWHM. Finally, the Bloch modes shown in Fig. 8(b) within the unit cell of the periodic structure disclose dipolar fields with enhanced localization at the boundary of the wires.

Figure 9 shows the intensity $\left|h_{x q}\right|^{2}$ evaluated by means of the integral transform in Eq. (25), which corresponds to nondiffracting beams propagating at a frequency $\beta=0.8 k_{0}$ in a wire medium of $f=0.1$ with Ag diameter $d=50 \mathrm{~nm}$. Subfigures (a) and (b) correspond to Fourier expansions using the branches $q=1$ and $q=2$ of the isofrequency curve shown in (a)

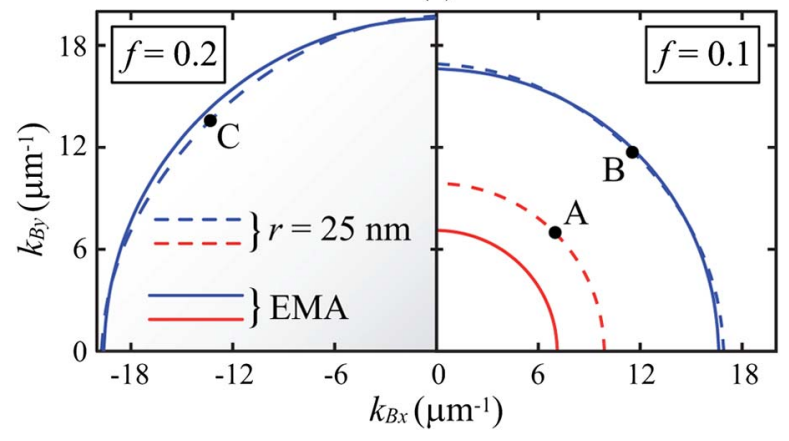

(b)
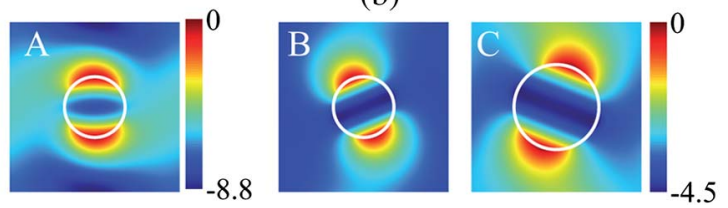

Fig. 8. (a) Isofrequency curve for the $\mathrm{Ag}^{-} \mathrm{Al}_{2} \mathrm{O}_{3}$ compound at a propagation constant $\beta=0.8 k_{0}$. The case of a wire diameter $d=$ $50 \mathrm{~nm}$ (dashed lines) is compared with EMA estimations (solid lines). (b) Intensity $\left|\mathbf{x} \cdot \mathbf{h}_{\mathbf{k}_{B}}\right|^{2}$ for Bloch modes at wave vectors set by the points A, B, and C. Scaling is set in $\mathrm{dB}$.

Fig. 8(a), respectively. Looking at the envelope of the wave field takes one back to the Bessel profiles depicted in Fig. 5, which are estimated by the EMA. Driven by SPPs, however, the wave field is clearly confined near the metal/dielectric interfaces. In addition, we put special emphasis on the beam compression of the nondiffracting beam for $q=1$ in comparison with the estimation given by the EMA.

If the FWHM of the diffraction-free beam reaches the diameter of the metallic cylinders in the squared array, the spatial distribution of the wave field varies significantly along with the origin of the coordinate system, $\mathbf{r}=(0,0)$. To illustrate this effect, Fig. 10 shows the intensity of the field $h_{x 2}$, which is evaluated by means of Eq. (25) and corresponds to nondiffracting beams evolving at an on-axis spatial frequency $\beta=0.8 k_{0}$ in our $\mathrm{Ag}-\mathrm{Al}_{2} \mathrm{O}_{3}$ compound. In this case, the metal filling fraction comes up to $f=0.2$, but the wire diameter $d=50 \mathrm{~nm}$ remains unaltered. When the origin is set at the center of a wire, the central spot exhibits a dipolar structure with a minimum of intensity at $\mathbf{r}=(0,0)$. However, if the origin is relocated at midpoint of two adjacent wires, the field is confined and intensified around the beam axis. Such a strong field enhancement has been widely exploited in gap optical antennas [39].

Inclusion of material losses also will modify the field pattern of the nondiffracting beam. In our case, this occurs if
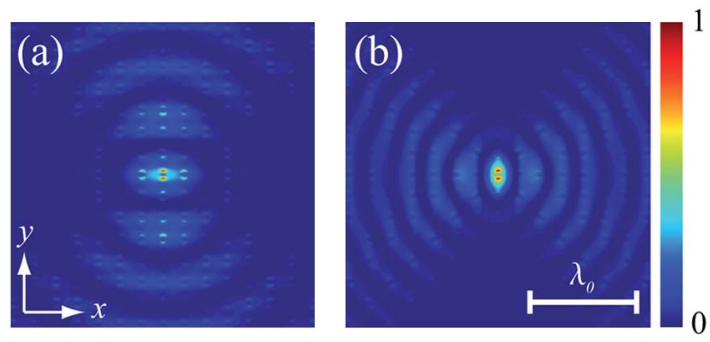

Fig. 9. Transverse profile of the intensity $\left|h_{x q}\right|^{2}$ corresponding to a nondiffracting beam of $\beta=0.8 k_{0}$ propagating in a wire medium of $f=0.1$ with cylinder diameter $d=50 \mathrm{~nm}$ in the cases: (a) $q=1$ and (b) $q=2$. 
(a)
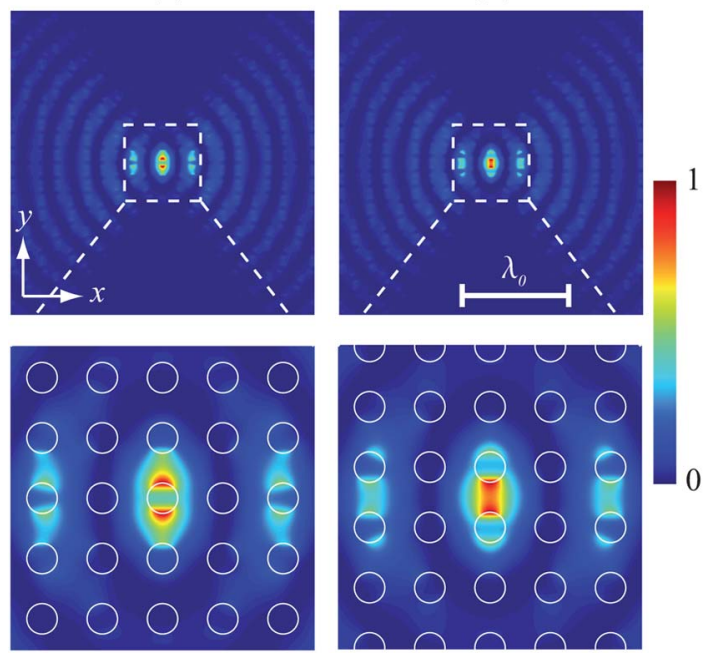

Fig. 10. Intensity $\left|h_{x 2}\right|^{2}$ of diffraction-free beams propagating at an on-axis spatial frequency $\beta=0.8 k_{0}$ in a Ag- $\mathrm{Al}_{2} \mathrm{O}_{3}$ compound of metal diameter $d=50 \mathrm{~nm}$ and filling fraction $f=0.2$. The origin $\mathbf{r}=(0,0)$ of the coordinate systems is set (a) at the center of a cylinder and (b) at midpoint of two adjacent wires. The field profile near the beam axis is scaled for the sake of clarity.

we consider the permittivity of silver to be complex, which is $\epsilon_{\mathrm{Ag}}=-20.4+i 1.29$ [36]. Let us now discuss the most relevant aspects concerning dissipation. Assuming that the magnetic field is expressed in terms of the Bloch modes given in Eq. (23), thus $\mathbf{k}_{B}$ continues to be a real valued wave vector. As a consequence, the propagation constant becomes complex, $\beta=\beta^{\prime}+i \beta^{\prime \prime}$, where $\beta^{\prime}$ and $\beta^{\prime \prime}$ stand for the real part and the imaginary part of the frequency $\beta$, respectively. Strictly speaking, nondiffracting beams rely on wave fields whose transverse pattern remains unaltered. Following the same procedure as above, the isofrequency curve in the $\mathbf{k}_{B}$ plane is evaluated given an on-axis spatial frequency $\beta^{\prime}$ in order to prevent defocusing. For an $\mathrm{Ag}-\mathrm{Al}_{2} \mathrm{O}_{3}$ compound of the same characteristics treated above $(f=0.2$ and $d=50 \mathrm{~nm})$, at $\lambda_{0}=700 \mathrm{~nm}$ and $\beta^{\prime}=0.8 k_{0}$, the changes determined in the isofrequency curve are negligible. Nevertheless, although the Bloch modes of the Fourier expansion in Eq. (25) propagate at the same spatial frequency along the $z$ axis, we point out that they experience a characteristic attenuation driven by a term $\exp \left(-\beta^{\prime \prime} \boldsymbol{z}\right)$.

In Fig. 11(a), we plot the on-axis intensity $\left|h_{x 2}\right|^{2}$ of a nondiffracting field whose beam axis is set at the center of a given wire; we have checked that the following conclusions will apply if the beam axis is displaced laterally. The exponential attenuation of the beam leads to a decrease in intensity of $44 \%$ at a propagation distance of one wavelength; it further undergoes a considerable reduction in intensity when travelling a distance of $5 \lambda_{0}$, remaining only at $8 \%$ of its starting intensity. Note also that the attenuation constant $\beta^{\prime \prime}$ varies in the modal expansion of Eq. (25). This means that some Bloch modes decay faster than others. As long as the beam propagates along the $z$ axis, the spatial spectrum of the beam undergoes significant changes leading to a transformation of its transverse profile. Figure 11(b) shows the waveform of the nondiffracting beam at different transverse planes. In practical terms, the nondiffracting nature of the beam is conserved before it is damped by silver losses, occurring within a (a)

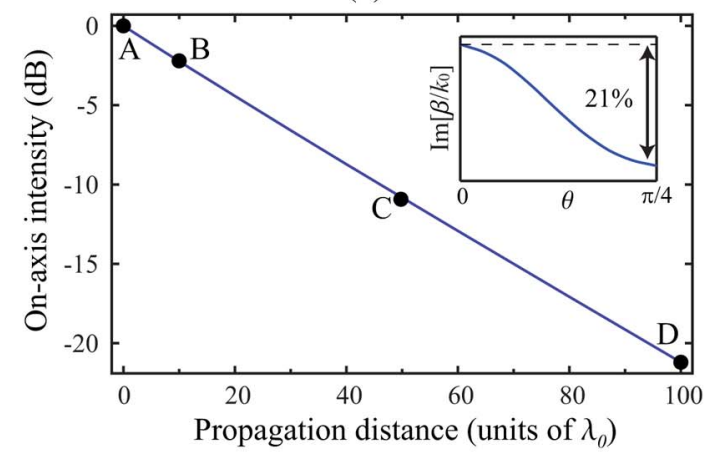

(b)
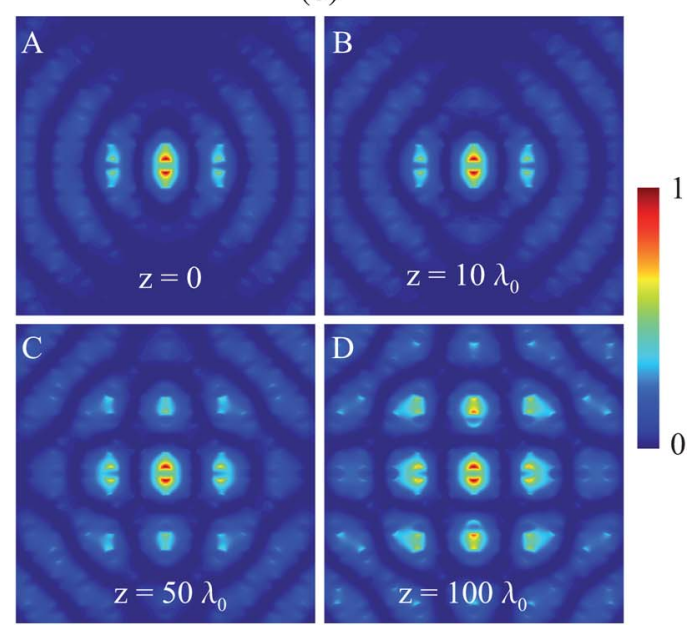

Fig. 11. (a) Normalized on-axis intensity if silver losses are included in the numerical simulation. In this case, $\beta=0.8 k_{0}$ in a Ag- $\mathrm{Al}_{2} \mathrm{O}_{3}$ compound of metal diameter $d=50 \mathrm{~nm}$ and filling fraction $f=0.2$. (b) Transverse profile of the wave field as the beam propagates along the $z$ axis.

few wavelengths. For a longer propagation distance, however, diffusion driven by dispersion in $\beta^{\prime \prime}$ induces blurring, thus breaking up wave confinement around the beam axis.

\section{CONCLUSIONS}

We presented a thorough discussion on diffraction-free localized beams propagating in metal-dielectric nanostructures. In this study, we considered a periodic squared distribution of wires at the nanoscale; however, more complex geometries might be treated similarly. We emphasize that the self-focusing mechanism under analysis differs from canalization in the metamaterial. Here, nondiffracting beams propagate with no distortion of its transverse pattern precisely due to the singular characteristics of the spatial distribution of this wave field.

For simplicity, first we considered a homogenization approach for the structured medium. We provided a general, analytical expression of the electromagnetic fields that can propagate along the optical axis of the homogenized composite, keeping its transverse waveform unaltered. The fields show Bessel waveforms and, set in a plane-wave Fourier expansion, represent a conical distribution of $o$ waves (TE modes) and $e$ waves (TM modes). We remark that TE BBs exhibit ultra-high spatial frequency, potentially featuring deep-subwavelength hot spots. In a numerical simulation, we reported a BB with central peak of FWHM as short as $\lambda_{0} / 8$. 
BBs in the hyperbolic wire medium were analyzed under TE polarization, but not for TM polarization. However, transverse profiles do not differ from those found in elliptic regimes. At most, higher propagation constants may be achieved for a given transverse spatial frequency. Beyond a limiting $k_{t}, e$ waves may only be supported in the medium, potentially leading to extremely high subwavelength beams.

Also we verified the validity of our analytical approach. The EMA showed good agreement with FEM simulations provided the wire diameter remains further down the penetration depth in the metal. When we considered an $\mathrm{Ag}-\mathrm{Al}_{2} \mathrm{O}_{3}$ composite with a wire diameter of $50 \mathrm{~nm}$, first we observed a significant compression of quasi-TM BBs. In general, the spatial distribution of the wave field also varied significantly along with the origin of the coordinate system. When the origins were set at midpoint of two wires gap, the field sharply concentrated around the beam axis. We concluded that composites with moderate $f$ help nonresonant plasmonic beams along propagating with a size that remains far below the limit of diffraction.

Finally, we demonstrated that inclusion of material losses will modify the field pattern of the nondiffracting beam. As long as the BB propagates, the spatial spectrum of the beam undergoes significant changes leading to a diffusive transformation of its transverse profile. In practical terms, however, the nondiffracting nature of the beam is conserved before it is damped by metal losses, occurring within a few wavelengths.

\section{ACKNOWLEDGMENT}

This research was funded by the Spanish Ministry of Economy and Competitiveness under the project TEC201129120-C05-01.

\section{REFERENCES}

1. J. W. Goodman, Introduction to Fourier Optics, 2nd ed. (McGraw-Hill International Editions, 1996).

2. M. Born and E. Wolf, Principles of Optics, 7th (expanded) ed. (Cambridge University, 1999).

3. A. E. Siegman, Lasers (University Science Books, 1986).

4. J. Durnin, "Exact solutions for nondiffracting beams. I. The scalar theory," J. Opt. Soc. Am. A 4, 651-654 (1987).

5. J. Durnin, J. J. Miceli, and J. H. Eberly, "Diffraction-free beams," Phys. Rev. Lett. 58, 1499-1501 (1987).

6. V. Garcés-Chávez, D. McGloin, H. Melville, W. Sibbett, and K. Dholakia, "Simultaneous micromanipulation in multiple planes using a self-reconstructing light beam," Nature 419, 145-147 (2002).

7. C. López-Mariscal and J. C. Gutiérrez-Vega, "Observation of optical guiding using thermal light," J. Opt. 12, 075702 (2010).

8. Y. Matsuoka, Y. Kizuka, and T. Inoue, "The characteristics of laser micro drilling using a Bessel beam," Appl. Phys. A 84, 423-430 (2006).

9. F. Courvoisier, P.-A. Lacourt, M. Jacquot, M. K. Bhuyan, L. Furfaro, and J. M. Dudley, "Surface nanoprocessing with nondiffracting femtosecond Bessel beams," Opt. Lett. 34, 3163-3165 (2009).

10. F. O. Fahrbach, V. Gurchenkov, K. Alessandri, P. Nassoy, and A. Rohrbach, "Light-sheet microscopy in thick media using scanned Bessel beams and two-photon fluorescence excitation," Opt. Express 21, 13824-13839 (2013).

11. A. Dudley, M. Lavery, M. Padgett, and A. Forbes, "Unraveling Bessel beams," Opt. Photon. News 24, 22-29 (2013).

12. M. Ornigotti and A. Aiello, "Radially and azimuthally polarized nonparaxial Bessel beams made simple," Opt. Express 21, 15530-15537 (2013).

13. S. Longhi, K. Janner, and P. Laporta, "Propagating pulsed Bessel beams in periodic media," J. Opt. B 6, 477-481 (2004).
14. O. Manela, M. Segev, and D. N. Christodoulides, "Nondiffracting beams in periodic media," Opt. Lett. 30, 2611-2613 (2005).

15. J. J. Miret and C. J. Zapata-Rodrguez, "Diffraction-free beams with elliptic Bessel envelope in periodic media," J. Opt. Soc. Am. B 25, 1-6 (2008).

16. S. N. Kurilkina, V. N. Belyi, and N. S. Kazak, "Transformation of high-order Bessel vortices in one-dimensional photonic crystals," J. Opt. 12, 015704 (2010).

17. J. Fagerholm, A. T. Friberg, J. Huttunen, D. P. Morgan, and M. M. Salomaa, "Angular-spectrum representation of nondiffracting $\mathrm{X}$ waves," Phys. Rev. E 54, 4347-4352 (1996).

18. Q. Zhan, "Evanescent Bessel beam generation via surface plasmon resonance excitation by a radially polarized beam," Opt. Lett. 31, 1726-1728 (2006).

19. W. Chen and Q. Zhan, "Realization of an evanescent Bessel beam via surface plasmon interference excited by a radially polarized beam," Opt. Lett. 34, 722-724 (2009).

20. A. Minovich, A. E. Klein, N. Janunts, T. Pertsch, D. N. Neshev, and Y. S. Kivshar, "Generation and near-field imaging of Airy surface plasmons," Phys. Rev. Lett 107, 116802 (2011).

21. P. Zhang, S. Wang, Y. Liu, X. Yin, C. Lu, Z. Chen, and X. Zhang, "Plasmonic Airy beams with dynamically controlled trajectories," Opt. Lett. 36, 3191-3193 (2011).

22. J. Lin, J. Dellinger, P. Genevet, B. Cluzel, F. de Fornel, and F. Capasso, "Cosine-Gauss plasmon beam: a localized longrange nondiffracting surface wave," Phys. Rev. Lett. 109, 093904 (2012).

23. J. J. Miret and C. J. Zapata-Rodríguez, "Diffraction-free propagation of subwavelength light beams in layered media," J. Opt. Soc. Am. B 27, 1435-1445 (2010).

24. C. J. Zapata-Rodríguez, S. Vuković, M. R. Belić, D. Pastor, and J. J. Miret, "Nondiffracting Bessel plasmons," Opt. Express 19, 19572-19581 (2011).

25. P. A. Belov and Y. Hao, "Subwavelength imaging at optical frequencies using a transmission device formed by a periodic layered metal-dielectric structure operating in the canalization regime," Phys. Rev. B 73, 113110 (2006).

26. J. J. Miret, D. Pastor, and C. J. Zapata-Rodríguez, "Subwavelength surface waves with zero diffraction," J. Nanophoton. 5, 051801 (2011).

27. C. R. Simovski, P. A. Belov, A. V. Atrashchenko, and Y. S. Kivshar, "Wire metamaterials: physics and applications," Adv. Mater. 24, 4229-4248 (2012).

28. P. A. Belov, R. Marques, S. I. Maslovski, I. S. Nefedov, M. Silveirinha, C. R. Simovski, and S. A. Tretyakov, "Strong spatial dispersion in wire media in the very large wavelength limit," Phys. Rev. B 67, 113103 (2003).

29. D. R. Smith and J. B. Pendry, "Homogenization of metamaterials by field averaging,” J. Opt. Soc. Am. B 23, 391-403 (2006).

30. D. Kim and S. J. Yoon, "Effective medium-based analysis of nanowire-mediated localized surface plasmon resonance," Appl. Opt. 46, 872-880 (2007).

31. A. Sihvola, Electromagnetic Mixing Formulas and Applications (Institution of Electrical Engineers, 1999).

32. A. A. Krokhin, P. Halevi, and J. Arriaga, "Long-wavelength limit (homogenization) for two-dimensional photonic crystals," Phys. Rev. B 65, 115208 (2002).

33. A. Ciattoni and C. Palma, "Nondiffracting beams in uniaxial media propagating orthogonally to the optical axis," Opt. Commun. 224, 175-183 (2003).

34. V. P. Drachev, V. A. Podolskiy, and A. V. Kildishev, "Hyperbolic metamaterials: new physics behind a classical problem," Opt. Express 21, 15048-15064 (2013).

35. G. B. Arfken and H. J. Weber, Mathematical Methods for Physicists (Academic, 2001).

36. E. D. Palik and G. Ghosh, The Electronic Handbook of Optical Constants of Solids (Academic, 1999).

37. K. Dolgaleva and R. W. Boyd, "Local-field effects in nanostructured photonic materials," Adv. Opt. Photon. 4, 1-77 (2012).

38. P. A. Belov, Y. Hao, and S. Sudhakaran, "Subwavelength microwave imaging using an array of parallel conducting wires as a lens," Phys. Rev. B 73, 033108 (2006).

39. P. Mühlschlegel, H.-J. Eisler, O. J. F. Martin, B. Hecht, and D. W. Pohl, "Resonant optical antennas," Science 308, 1607-1609 (2005). 